\title{
A Study of Seismic Macroeconomic Losses Based on Monte Carlo Method-Take Tangshan City as an Example
}

\author{
Qing Wu* ${ }^{*}$ Mengtan Gao \\ Institute of Geophysics, China Earthquake Administration, Beijing, 100081, China
}

Received October 26, 2018

Accepted November 10, 2018

\begin{abstract}
The Monte Carlo method is used to simulate seismic sequences. For each earthquake in the sequence, the ground motion parameters of each site are calculated by the attenuation relationship, which is introduced into the vulnerability of the macroeconomic with GDP loss as an indicator. The model gives the exceeding probability curve of macroeconomic losses. This method provides a straightforward way to estimate the level of economic loss of a city in future earthquakes. It is of great importance to decision-making reference for formulating the corresponding earthquake prevention and mitigation countermeasures at present stage, and also provides a reference for insurance companies to determine the rates.
\end{abstract}

Keywords: Monte Carlo method, macroeconomic vulnerability model, exceeding probability curve

\section{基于蒙特卡罗方法的地震宏观经济损失研究一以唐山市为例}

\author{
吴 清*，高孟潭
}

1.中国地震局地球物理研究所, 北京 100081

\begin{abstract}
摘 要: 本文利用蒙特卡罗方法模拟地震序列, 对于地震序列里的每一个地震通过衰减关系计算得到各场 点地震动参数值, 带入到以国内生产总值 GDP 损失为指标的宏观经济易损性模型, 给出了地震宏观经济损 失超越概率曲线。此方法为估计一个城市在未来地震中的经济损失水平提供了一种简单直接的思路, 对于 在现阶段制订相应的防震减灾对策具有十分重大的决策参考意义，更为保险公司厘定费率提供借鉴。
\end{abstract}

关键词：蒙特卡罗方法; 宏观经济易损性模型; 超越概率曲线

\section{1. 引言}

灾害定量化是地球科学、工程科学和社会经济 科学的交叉领域。人口城市化是全球的发展趋势。现 代城市在抗御自然灾害方面, 比过去任何时候都显得 更为脆弱 ${ }^{[1]}$ 。城市化在给人类带来富裕的物质生活和 丰富多彩的文化生活的同时, 也会因人口、财富在有 限空间的高度集中, 造成维系城市正常运转的基础设

*通讯作者：吴清(wuqing908@sina.com)
施、生命线工程、建筑物的日益庞大和复杂。尤其是 GDP值高的城市, 经济发达、人口稠密, 单位面积的 社会财富远远高于全国的平均水平。一旦这些重要城 市遭受大地震袭击, 将会造成重大的人员伤亡和巨额 的经济损失，甚至会对整个国家的经济发展造成巨大 冲击。我国许多大城市和城市群位于易发生大地震的 大型活动断裂带附近, 其中包括 12 个省会城市。改 革开放以来, 大量的人口、资产和重大设施快速向这 些城市集中。暴露在具备发生 7 级以上大地震的危险 区域的人口、资产以指数方式迅速累积, 导致地震风 
险塌升 ${ }^{[2]}$ 。如何分析一个城市在未来地震中的经济损 失水平, 对于在现阶段采取相应的防震减灾对策具有 重大的决策参考意义。

传统的地震灾害损失研究通常采用易损性分类 清单方法, 要求对研究区内建筑设施建立分类数据 库, 并详尽的收集资料用以建立震害矩阵。但面对现 代社会的快速发展, 使得传统的方法在收集并及时更 新资料方面都存在着明显的局限性, 而这种详细的分 类资料在许多地区甚至是难以获得的。陈颗等 ${ }^{[3]}$ 在执 行联合国 “国际减轻自然灾害十年” 计划中论证了可 以将承灾体脆弱性转化为国民生产总值 (GDP) 脆弱 性, GDP资料容易获得且更新及时, 随后陈颙等 ${ }^{[4-5]}$ 、 陈棋福等 ${ }^{[7-8]}$ 、米宏亮 ${ }^{[9]}$ 建立了适用于不同尺度和地区 的以GDP损失为指标的宏观经济易损性模型。刘吉夫 等 ${ }^{[10-12]}$ 在此思路上开展了地震灾害损失预测研究, 完 善了该分析方法的人口和GDP分配方案, 以烈度区为 分配单位, 建立了地震宏观风险分析模型。王晓青等 [13]将此方法用于汶川地震损失快速评估中, 其结果与 袁一凡等 ${ }^{[14]}$ 进行现场调查得出的经济损失结果进行 了比较, 结果较为一致。杨斌等 ${ }^{[15]}$, 范志伟等 ${ }^{[16]}$ 都通 过实际震例验证了王晓青等 ${ }^{[13]}$ 提出的宏观经济易损 性模型在小尺度空间范围内震后经济损失快速评估 中的可行性。

过去的震害损失预测分析, 一般在考虑地震危险 性时是有概率水平的, 可是在分析震害及经济损失 时, 没有充分考虑这些因素的概率特征。刘本玉等 ${ }^{[17]}$ 以全概率的公式为基础, 建立了区域震害经济损失预 测的概率分析模型, 综合考虑了地震、场地、结构和 当地经济发展水平的影响。但是在公式推导过程中, 由于各因素之间的关系很难得到, 而做了许多简化与 平均, 在实际应用中比较难以实现与推广。

蒙特卡罗方法则是一种基于合成地震序列的地 震危险性计算方法, 该方法根据地震活动性模型可以 生成满足某一区域内地震时空分布规律的地震序列, 对地震序列里的每一个地震通过衰减关系计算得到 各场点地震动参数值, 进一步带入易损性模型, 可以 对地震风险进行评估 ${ }^{[18-23]}$ 。本文拟将蒙特卡罗方法与 宏观经济易损性模型结合起来, 将蒙特卡罗方法模拟 计算得到的地震事件影响场带入到以国内生产总值 GDP 损失为指标的宏观经济易损性模型, 给出地震 宏观经济损失超越概率曲线。

\section{2. 方法介绍}

蒙特卡罗 (Monte Carlo) 方法是一种应用随机数 来进行计算机模拟的方法。此方法对研究的系统进行 随机观察抽样, 通过对样本值的观察统计, 求得所研 究系统的某些参数。其基本思想是当实验次数充分多
时, 某一事件出现的频率近似于该事件发生的概率, 即

$$
\begin{aligned}
& \frac{x}{X} \approx p \text { 错误! 未找到引用源。（当 } \mathrm{X} \text { 充 } \\
& \text { 分大时) (1) }
\end{aligned}
$$

式中 $p$ 是某一事件发生的概率; $X$ 是实验次数; $X$ 是 在 $X$ 次实验中该事件出现的频次。

中国地震动参数区划图（GB18306-2015）依据 各个地区的地球物理资料, 划分了地震带和潜在震源 区, 建立了相宜的地震发生概率模型和空间分布模 型, 并给出了各个地震带的基本参数。根据中国地震 动参数区划图的基本假定和地震活动性参数, 可采用 以下步骤合成地震事件集 ${ }^{[22]}$ :

（1）基于地震带内地震发生满足泊松分布的假 定, 首先确定所要模拟地震序列的时间长度 $\mathrm{T}$ 和地震 带内地震的年平均发生率 $v_{4}$ 。随机产生一个以 $\mathrm{T}$ 和 $v_{4}$ 为参数的泊松分布随机数 $\mathrm{R}$, 那么 $\mathrm{R}$ 就是所要模 拟时间长度为 $\mathrm{T}$ 的该地震带上地震的数量。

（2）基于地震带内地震活动的震级分布满足截 断的 Gutenberg-Richter 关系（震级-频度关系）的假 定, 以及最小震级水平 $M_{0}$ 和最大震级上限 $M_{U z}$ 来确 定震级。

$$
\begin{array}{r}
\text { 震级-频度关系表达式为: } \\
\operatorname{logN}=a-b M
\end{array}
$$

式中, $a, b$ 为系数, $N$ 为震级大于等于 $M$ 的地震个数, $M=4,4.1,4.2, \cdots, M$, 中国地震动参数区划图的起算 震级为 4 级。则地震事件累积数量的值为:

$$
N(\mathrm{M})=\mathrm{e}^{a-b M}
$$

取 $\Delta M=0.1$, 则有

$$
N(\mathrm{M})>\mathrm{N}(\mathrm{M}+\Delta \mathrm{M})
$$

随机生成一个符合在 0 和 1 之间均匀分布的随 机数, 判断它是否在 $\frac{N(\mathrm{M}+\Delta \mathrm{M})}{N(4)}$ 错误! 未找到引用源。 与 $\frac{N(\mathrm{M})}{N(4)}$ 之间, 如果是, 则确定地震事件的震级 $M$ 。

（3）震中位置的确定。首先, 确定地震事件所 在的潜在震源区 $H$ 。根据上步所确定的震级 $M$ 判定 地震所在的震级档 $d$, 因为每个震级档的地震落在各 个潜在震源区的概率 $P_{d}(\mathrm{~h})$ 是已知的并且是归 1 的, 那么随机产生一个符合在 0 和 1 之间均匀分布的随机 
数, 判断它在是否在 $\sum_{\mathrm{h}=1}^{H} P_{d}(\mathrm{~h})$ 错误! 未找到引用源。 与 $\sum_{\mathrm{h}=1}^{H-1} P_{d}(\mathrm{~h})$ 之间, 如果是, 则确定地震事件所在的潜 在震源区 $\mathrm{H}$ 。基于震中在潜在震源区内均匀分布的假 定，随机抽取一点作为地震的震中位置。

（4）根据潜在震源区衰减长轴方位角的概率, 抽 样确定地震方位角 $\theta$ 。

至此, 确定了一个地震的基本要素。重复 (2) (4) 步直至所需的该地震带上的地震数目 $R$, 综合 考虑所有可能对场点造成影响的地震带, 由此确定出 来一个地震序列, 完成一次抽样。通过大规模抽样模 拟，形成地震概率事件集。

时间长度若设置为 1 年, 则完成一次抽样模拟得 到的地震序列本文称为 1 年地震序列。时间长度设置 为 10 年, 即称为 10 年地震序列。

根据蒙特卡罗方法原理, 抽样次数越多结果越精 确, 但抽样次数增多会显著增加计算量。因此, 为了 统筹考虑计算量和结果精确程度, 需进行不同抽样次 数的试验, 当计算结果趋于稳定时, 即认为不再需要 增加抽样次数。

对于概率事件集里的每一个地震, 以地震的经纬 度为原点, 衰减长轴方向为正方向, 将所有场点转换 到平面坐标下。根据中国地震动参数区划图的椭圆烈 度衰减关系, 采用最优椭圆搜索的数值计算方法计算 地震在各个场点处的烈度值 I。

宏观经济易损性模型可表示为:

$$
F(\mathrm{I}, \mathrm{GDP})=\mathrm{C} \cdot \mathrm{A} \cdot \mathrm{I}^{\mathrm{B}}
$$

式中, F(I,GDP)代表 GDP 损失率, I 为地震烈度, A、 $B$ 为系数, $C$ 为修正系数。 $A 、 B$ 值直接从回归关系 式获得, 修正系数 C一般取 1.0。

对于一个行政区域（市）, 将其所辖的区县或乡 镇各自视为一个场点, 每个场点附有各自的 GDP 值。 对于用蒙特卡罗方法模拟得到的地震序列里的每一 个地震, 通过烈度衰减关系可以同时计算得到所有场 点的影响烈度值 $I_{i}$, 带入宏观易损性模型, 可以计算 得到这个地震对行政区域可能造成的损失:

$$
\text { LOSS }=\sum_{i=1}^{j} C \cdot A \cdot I_{i}^{B} \cdot G D P_{i}
$$

式中 $j$ 是区县或乡镇总数。这样地震序列里每一个地 震对应一个 GDP 损失值, 选取序列里的最大值作为 一次模拟的结果。随机模拟 $X$ 次, 得到 $X$ 个地震序
列, 统计超越给定经济阈值的相应地震序列个数 $x$, 计算得到超越概率值。

在计算过程中需考虑经济及社会财富的动态发 展, 即 GDP 值的增长变化。如果以当前资料搜集年 的 GDP 产值为基础, 用简单的年均增长率 $q$ 来考虑 的话，则第 $k$ 年的国内生产总值为 $G D P \times(1+q)^{k-1}$, 如此 $T$ 年内的 GDP 总值为

$$
Y=\sum_{k=1}^{T} G D P \times(1+q)^{k-1}=G D P \times \frac{(1+q)^{T}-1}{q}
$$

则在 $T$ 年内以 GDP 描述的社会平均财富为

$$
\overline{G D P}=Y / T
$$

将之代替损失公式中的 GDP 值。

本文收集整理了自 1989 年以来, 开展正式地震 现场震害调查和损失评估的中国大陆地震的灾害损 失资料, 拟回归宏观经济易损性模型的系数。但由于 收集到的灾评报告基本以划分的评估区或者行政区 为单位给出经济损失估计值，而评估区和行政区往往 与烈度区并不重合, 导致经济损失和人员伤亡有时无 法归算到烈度区。而宏观经济易损性模型的高烈度部 分是根据拟合公式外推的, 因此需要更多的大地震震 例来验证。

王晓青等 ${ }^{[13]}$ 给出了宏观经济易损性模型:

$$
F(\mathrm{I}, \mathrm{GDP})=\left\{\begin{array}{lr}
2 \times 10^{-8} \cdot I^{9.8082} & \text { 人均 } G D P<2700 \\
2 \times 10^{-10} \cdot I^{11.585} & \leq \\
4 \times 10^{-11} \cdot I^{11.377} & \text { 人均 } G D P<10000
\end{array}\right.
$$

该模型用于汶川地震损失快速评估中, 其结果与 袁一凡等 ${ }^{14}$ 进行现场调查得出的经济损失结果进行 了比较, 结果较为一致。杨斌等 ${ }^{[15]}$, 范志伟等 ${ }^{[16]}$ 都通 过实际震例验证了王晓青等 ${ }^{[13]}$ 提出的宏观经济易损 性模型在小尺度空间范围内的可行性。因此, 本文也 借鉴这一模型进行计算。

\section{3. 计算实例}

1976 年 7 月 28 日, 唐山发生了举世震惊的 7.8 级强烈地震，使百年工业城市瞬间毁于一旦。地震造 成 24.2 万人死亡, 16.4 万人重伤, 铁路交通、邮电 通信、供水供电完全中断, 道路桥梁、工厂设备和农 田水利设施遭到严重破坏, 直接经济损失高达 54 亿 元。灾情之重、损失之巨，举世罕见。经过 10 年的 恢复重建和 30 年的振兴发展, 今天的唐山已然成为 一个现代化新型都市, 成为国际灾后城建的典范。然 而唐山依然处于发震构造带上, 其地震风险随着唐山 
市经济的高速发展和人口密度的集中高度殺升, 甚至 远超 40 年前。本文拟通过蒙特卡罗方法给出唐山市 $\mathrm{T}$ 年内地震宏观经济损失超越概率曲线, 为城市减灾 战略规划需要和地震应急需求提供参考。

图 1(a)为唐山市行政区域分布图, 将 18 个区县 各自视为一个场点, 每个场点附有各自的 GDP 值;

图 1(b)是唐山地区周边地震带分布图; 图 1(c)是覆盖 唐山地区的潜在震源区分布图; 图 1(d)为唐山地区蒙 特卡罗方法 1 年地震序列模拟 100000 次中的 $5.0 \leqslant$ $\mathrm{M}<8.0$ 的地震震中分布示意图。表 1 是计算所用到的 中国地震动参数区划图中地震带的地震活动性参数 列表; 表 2 是 2016 年唐山市各区县 GDP 值 (当年价) 和年增长率 ${ }^{[24]}$ 。

利用GB18306-2015 中国地震动参数区划图华北 地区椭圆烈度衰减模型, 按最优椭圆搜索法逐次计算 地震序列里的每一个地震在 18 个场点处的烈度值I。 华北地区烈度椭圆衰减关系如下 ${ }^{[25]}$ :

长轴方向

$$
I=5.7123+1.3626 \cdot M-4.2903 \cdot \lg (\mathrm{R}+25) \quad(10)
$$

短轴方向

$$
\begin{gathered}
I=3.6588+1.3626 \cdot M-3.5406 \cdot \lg (\mathrm{R}+13) \\
\sigma=0.5826
\end{gathered}
$$

式中, $I$ 为烈度, $M$ 为震级, $R$ 为震中距, $\sigma$ 为 标准差。

由于蒙特卡罗方法抽样次数越多结果越精确, 为 了统筹考虑计算量和结果精确程度, 分别进行了 1 年 地震序列的 10 万次、 100 万次、 1000 万次以及 10 年 地震序列的 10 万次和 100 万次抽样计算，统计不同 模拟次数下和不同时间长度的经济损失超越概率, 结 果见图 2。由图 2 可以见到, 对于 1 年地震序列, 1000 万次的模拟足以给出稳定平滑的年超越概率曲线; 而 对于 10 年时间长度的地震序列, 100 万次的模拟也 呈现稳定平稳的超越概率曲线。

由超越概率曲线可以看到, 以 2016 年的经济水 平为基础, 唐山市 100 亿元地震经济损失的年超越概 率约为 $2 \%, 1000$ 亿地震经济损失的年超越概率约为 $0.38 \%, 10000$ 亿地震经济损失的年超越概率约为
$0.02 \%, 10$ 年的宏观经济损失超越概率也符合概率计 算规律。

表 1 计算所用到的地震区带地震活动性参数列表

\begin{tabular}{ccccc}
\hline 地震区带名称 & $\mathrm{b}$ 值 & $v_{4}$ & $M_{u z}$ & 潜在震源区数目 \\
\hline 怣庐地震带 & 0.85 & 4.0 & 8.5 & 107 \\
华北平原地震带 & 0.86 & 4.6 & 8.0 & 97 \\
汾渭地震带 & 0.78 & 2.5 & 8.5 & 57 \\
东北地震区 & 1.00 & 5.0 & 7.5 & 117 \\
\hline
\end{tabular}

表 22016 年唐山市各区县生产总值 (GDP)

\begin{tabular}{ccc}
\hline 县 (市) 区 & 地区生产总值 (亿元) & 累计增长\% \\
\hline 全市 & 6354.87 & 6.8 \\
曹妃甸区 & 367.88 & 7.2 \\
海港开发区 & 130.59 & 8.5 \\
芦台开发区 & 43.81 & 12.6 \\
汉沽管理区 & 32.09 & 10.0 \\
路南区 & 123.10 & 7.5 \\
路北区 & 152.05 & 7.0 \\
古治区 & 193.26 & 4.3 \\
开平区 & 125.55 & 6.8 \\
丰南区 & 619.36 & 6.9 \\
丰润区 & 641.97 & 4.6 \\
高新开发区 & 126.07 & 8.0 \\
迁安市 & 920.16 & 3.7 \\
遵化市 & 512.24 & 5.9 \\
滦 县 & 462.07 & 6.2 \\
㴒南县 & 336.55 & 6.1 \\
乐亭县 & 345.18 & 7.6 \\
迁西县 & 425.97 & 6.5 \\
玉田县 & 380.91 & 5.8 \\
\hline
\end{tabular}




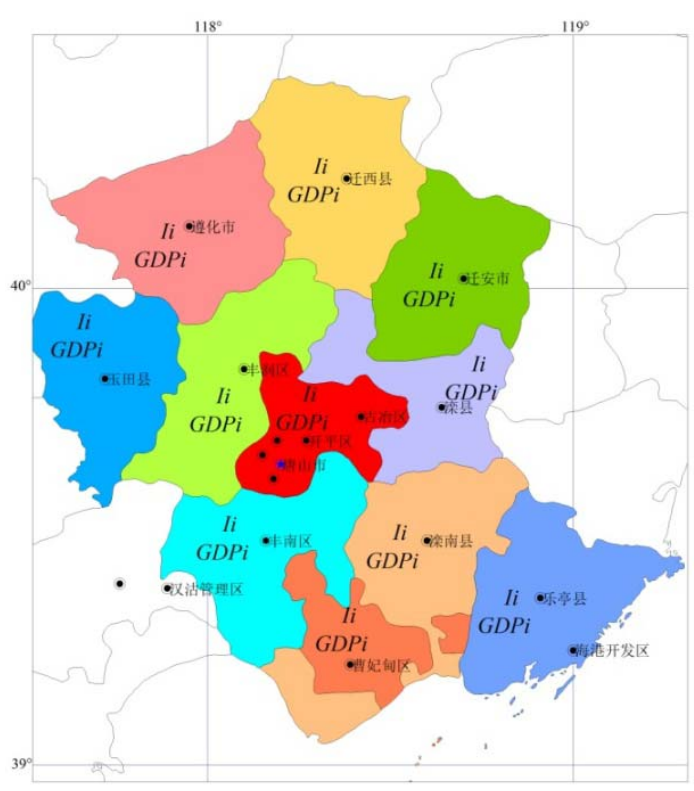

图 1(a) 唐山市行政区域分布图

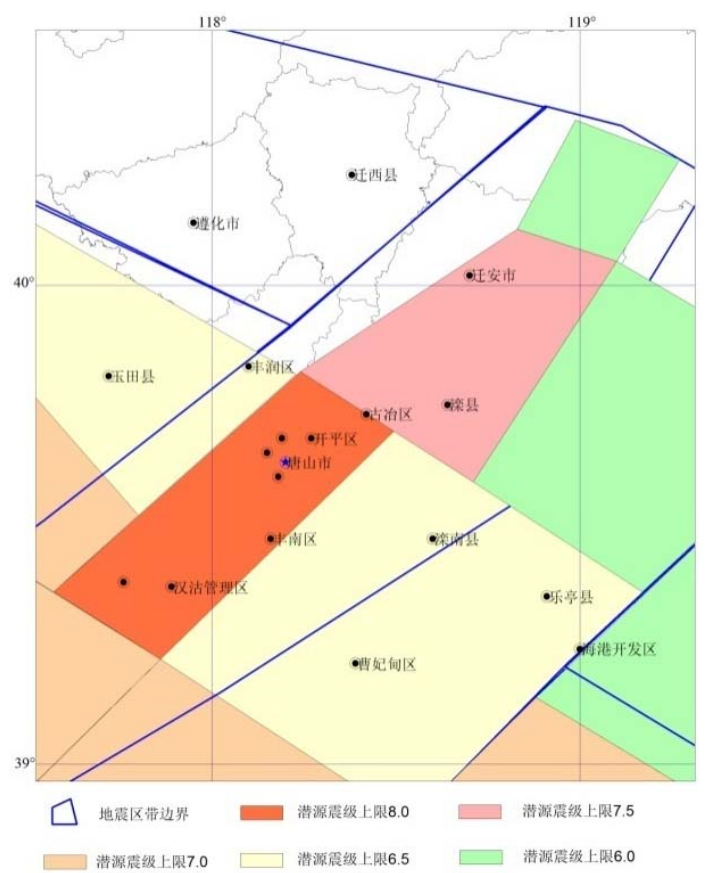

图 1(c) 唐山地区潜在震源区分布图

\section{4. 结论与讨论}

基于中国地震动参数区划图（GB18306-2015）, 利用蒙特卡罗方法建立概率地震事件集和地震动影 响场, 带入到宏观经济易损性模型, 简单直接的统计

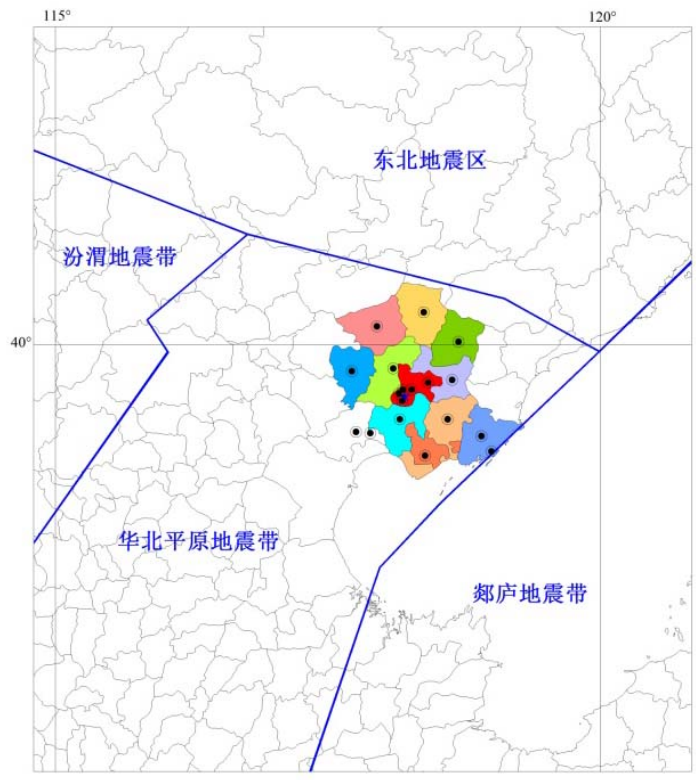

图 1(b) 唐山地区及周边地震带分布图

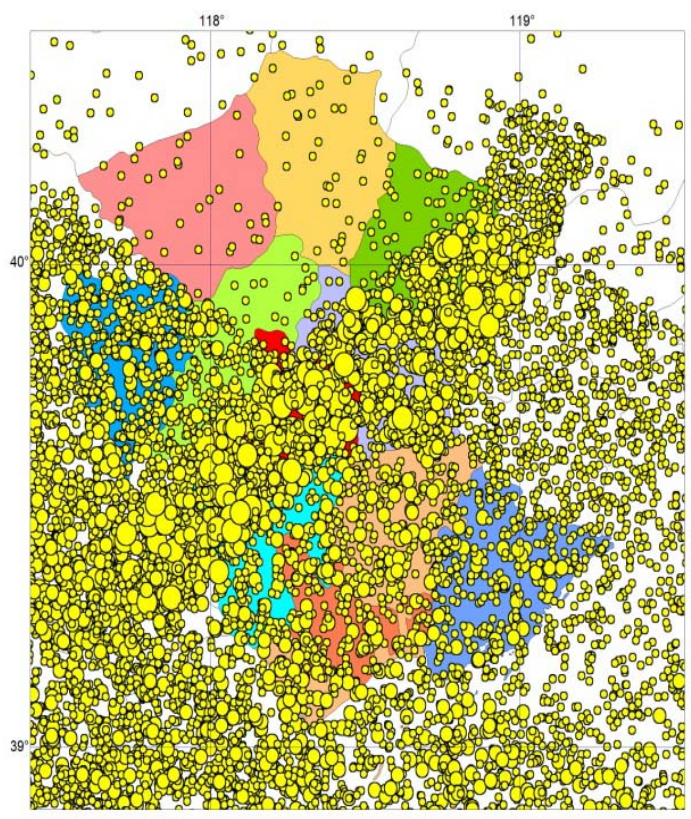

震级: $\bigcirc 7.0 \leq \mathrm{M}<8.0 \quad 06.0 \leq \mathrm{M}<7.0 \quad 05.0 \leq \mathrm{M}<6.0$

图 1(d)唐山地区 1 年地震序列模拟 100000 次中 $5.0 \leqslant$ $\mathrm{M}<8.0$ 的地震震中分布示意图

归算未来一段时间内城市经济损失水平的超越概率。 通过大规模模拟抽样, 以 2016 年经济水平为基础, 得到唐山市未来可能面临的地震经济损失超越概率 曲线, 此方法给地震经济损失估计赋予了概率的意 义。 


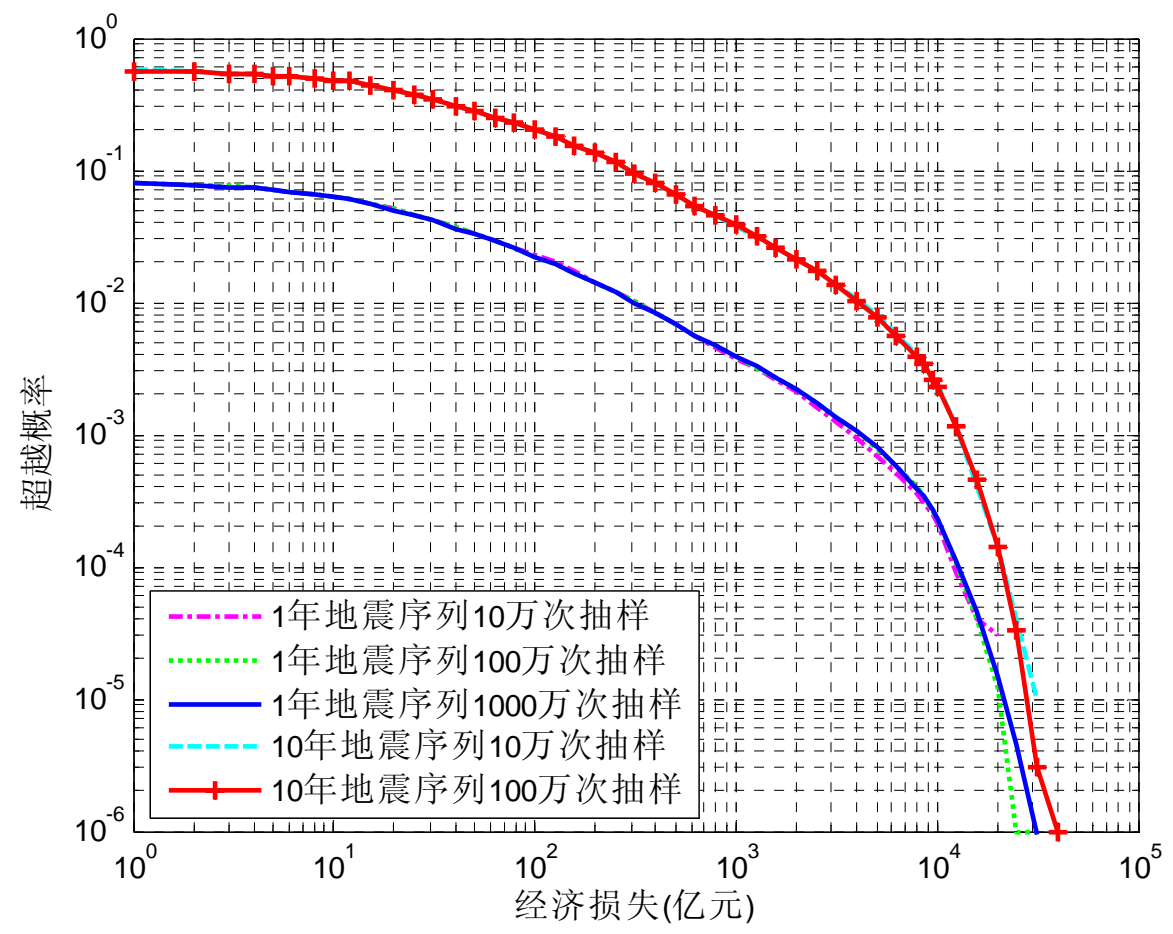

图 2 宏观经济损失超越概率曲线 new generation of seismic zonation map of

由于蒙特卡罗方法是逐次计算每个模拟地震可 能对城市造成的经济损失, 那么很容易找到给城市造 成最大损失的那些地震, 进而构建更为详细的地震情 景, 以更精确的估计它们可能的影响。

蒙特卡罗方法原理简单直接, 可以很容易被规划 者、施政者和保险公司理解接受。以此方法来估计一 个城市在未来地震中的经济损失水平, 对于在现阶段 制订相应的防震减灾对策具有十分重大的决策参考 意义，更为保险公司厘定费率提供借鉴。

\section{致谢}

This study was supported by Major Research Plan of Institute of Geophysics, China Earthquake Administration, Basal Research Fund (DQJB17C08).

\section{参考文献}

[1] C. Yong, P. Wengtao, X. Wenli, New Feature of Earthquake Disaster in 21st Century, Advance in earth sciences, 19(3) (2004) 359-363.

陈颙,彭文涛,徐文立, 21 世纪地震灾害的一些新特点 $[\mathrm{J}]$, 地球科学进展,19(3),2004:359-363. and Disaster Reduction, (3) (2016) 1-5.

高孟潭, 新一代国家地震区划图与国家社会经济发展, 城市与减灾,(3),2016:1-5.

[3] C. Yong, Z. Hongren, Research on Quantification of Earthquake Disasters, Recent Developments in World Seismology, (5) (1991) 5-9.

陈颙, 朱宏任. 地震灾害定量化研究. 国际地震动态, (5), 1991:5-9.

[4] C. Yong, C. Qifu, C. Ling, Vulnerability Analysis in Earthquake Loss Estimate, Earthquake Research in China, 15(2) (1999) 97-105.

陈颙,陈棋福,陈凌, 地震损失预测评估中的易损性分析, 中国地震,15(2),1999:97-105.

[5] Chen Yong, Chen Qifu, Chen Ling, 2001, Vulnerability analysis in earthquake loss estimate, Natural Hazards, (16):1-16.

[6] Chen Yong , Chen Qifu, Liu Jie , et al ., 2002,Seismic Hazard and Risk Analysis : A Simplified Approach . Beijing, Science Press.

[7] C. Qifu, C. Yong, C. Ling, Forecast and Assessment of Earthquake Disaster Losses Using Gross Domestic Product and Population Data, Acta Seismologica Sinica, 19(6) (1997) 640-649.

陈棋福, 陈颙, 陈凌, 利用国内生产总值和人口数据进行 地震灾害损失预测评估,地震学报,19(6),1997:640-649.

[8] C. Qifu, W. Xiaoqing, L. Jifu, Study on Seismic Vulnerability of Macroeconomic Indicators (GDP) in Mainland China, Prediction of earthquake hazard zone and earthquake disaster loss in Mainland China from 2006 to 2020, Beijing, Seismological Press, 2007. 
陈棋福,王晓青,刘吉夫,中国大陆宏观经济指标(GDP)的 地震易损性研究[A].2006-2020 年中国大陆地震危险 区与地震灾害损失预测研究编委会.2006-2020年中国 大陆地震危险区与地震灾害损失预测研究[C].北京:地 震出版社,2007:252-264.

[9] M. Hongliang, Research on Macro-vulnerability of Earthquake Disasters and Network Realization of Rapid Assessment of Earthquake Disasters, M.S. thesis, Beijing: the Center for Analysis and Prediction, China Earthquake Administration, (2002), pp. 19-48.

米宏亮, 地震灾害宏观易损性研究与震害快速评估的网 络实现 [D]. 硕士论文, 中国地震局分析预报中 心,2002,19-48.

[10]L. Jifu, The Study on Applicability of Macroscopic Indicator Vulnerability to a Small-Scale Region, PhD thesis, Beijing: Institute of Geophysics, China Earthquake Administration, (2006), pp. 21-38.

刘吉夫,2006,宏观震害预测方法在小尺度空间上的适 用性研究 [D]. 博士论文, 中国地震局地球物理研究 所,21-38.

[11]L. Jifu, C. Yong, S. Peijun, et al, On Seismic Risk Assessment in Mainland China, Journal of Beijing Normal University (Natural Science), 44(5) (2008) 520-523.

刘吉夫,陈颙,史培军等,2008,中国大陆地震风险分析模 型研究, 北京师范大学学报(自然科学版),44(5):520-523.

[12]L. Jifu, C. Yong, S. Peijun, et al, A Study on Seismic Risk Assessment in Mainland China( II ): Life Vulnerability Model, Journal of Beijing Normal University (Natural Science), 44(4) (2009) 404-407.

刘吉夫,陈颙,史培军等,2009, 中国大陆地震风险分析模 型研究( II ): 生命易损性模型, 北京师范大学学报(自然 科学版),45(4):404-407.

[13]W. Xiaoqing, D. Xiang, W. Long, et al, A Study on Fast Earthquake Loss Assessment and Its Application to 2008 Wenchuan M8 Earthquake, Acta Seismologica Sinica, 31(2) (2009) 205-211.

王晓青,丁香,王龙等,四川汶川 8 级大地震灾害损失快 速评估研究[J].地震学报,2009,31(2):205-211.

[14]Y. Yifan, Loss Assessment of Wenchuan Earthquake, Journal of Earthquake Engineering and Engineering Vibration, 28(5) (2008) 10-19.

袁一凡,四川汶川 8.0 级地震损失评估[J].地震工程与工 程振动,28(5),2008:10-19.

[15]Y. Bin, M. Zhaohui, The Application of the Disaster Losses Macroscopic Forecasting Model in the Rapid Assessment after Earthquake, Seismological and Geomagnetic Observation and Research, 34(3/4)(2013) 213-216.

杨斌,马朝晖,灾害损失宏观预测模型震后快速评估,地 震地磁观测与研究,34(3/4),2013:213-216.

[16]F. Zhiwei, Y. Fan, C. Xiaoyan, Economic Loss of Rapid Assessment after Earthquake in Zhangjiakou, Journal of Disaster Prevention and Reduction, 32(3) (2016) 15-18. 范志伟,杨凡,陈晓燕,基于宏观经济指标对张家口地区 地震灾后经济损失快速评估,防灾减灾学报, 32(3), 2016:15-18.
[17]L. Benyu, S. Jingyu, J. Jianjing, et al, Total Probability Forecasting Method for Economic Loss of Seismic Damage, World Earthquake Engineering, 19(1) (2003) 15-20.

刘本玉, 苏经宇, 江见鲸, 李刚, 震害经济损失的全概率预 测,世界地震工程,19(1),2003:15-20.

[18]Gao Mengtan, Pan Hua, Characteristics of Random Field for Seismic Zoning Results. Acta Eismologica Sinica, 15(1) (1993) 53-60.

高孟潭, 潘华, 地震区划结果随机场特征 [J], 地震学 报,15(1),1993:53-60.

[19]Musson, R. M. W. 1999, Determination of design earthquakes in seismic hazard analysis through Monte Carlo simulation, J. Earthquake. Eng., Vol.3, 463-474.

[20]Musson, R.M.W., 2000, The use of Monte Carlo simulations for seismic hazard assessment in the U.K., Annaili Di Geofisica, 43(1): 1-9.

[21]Smith, W. D., 2003, Earthquake Hazard and Risk Assessment in New Zealand by Monte Carlo Methods, Seismological Research Letters, 74(3): 298-304.

[22]G. Xing, Probabilistic Seismic hazard assessment based on Monte Carlo simulation, M. S. thesis, Beijing: Institute of Geophysics, China Earthquake Administration, (2008), pp. 16-18.

郭星,2008, 基于蒙特卡罗模拟的概率地震危险性分析 方法,中国地震局地球物理研究所硕士论文,16-18.

[23]C. Kaufmann, J. Schwarz,2014, Earthquake hazard and risk assessment tool using Monte-Carlo simulation techniques, Tenth U.S. National Conference on Earthquake Engineering Frontiers of Earthquake Engineering July 21-25,Anchorage, Alaska.

[24]Tangshan Statistical Bureau, Tangshan Investigation Team of National Statistical Bureau, Tangshan Statistical Summary 2016, Hebei: Printing Factory of Tangshan Municipal Committee of the Communist Party of China, 2017, 46.

唐山市统计局, 国家统计局唐山调查队,2017, 唐山统计 概要 2016,河北:中共唐山市委机关印刷厂,46.

[25]X. Liang, Study on the Attenuation Relationships of Horizontal Ground Motion Parameters Near the Source of Rock Site, PhD thesis, Beijing: Institute of Geophysics, China Earthquake Administration, (2011), pp. 104. 肖亮, 水平向基岩强地面运动参数衰减关系研究[D], 北 京:中国地震局地球物理研究所,2011,104. 